Rev. bras. paleontol. 15(2):153-163, Maio/Agosto 2012

(C) 2012 by the Sociedade Brasileira de Paleontologia

doi:10.4072/rbp.2012.2.04

\title{
ICNOFÓSSEIS DA BACIA DO PARNAÍBA: AS CONTRIBUIÇÕES DE WILHELM KEGEL
}

ANTONIO CARLOS SEQUEIRA FERNANDES, VERA MARIA MEDINA DA FONSECA

Departamento de Geologia e Paleontologia, Museu Nacional, Universidade Federal do Rio de Janeiro/UFRJ, Quinta da Boa Vista s/n, São Cristóvão, 20940-040, Rio de Janeiro, RJ.fernande@acd.ufrj.br,vmmedinafonseca@gmail.com

LUIZA CORRAL MARTINS DE OLIVEIRA PONCIANO

Departamento de Geologia, IGEO/UFRJ, Av. Athos da Silveira Ramos, 274, CCMN, Cidade Universitária, 21941-916, Rio de Janeiro, RJ. luizaponciano@gmail.com

\begin{abstract}
ICHNOFOSSILS FROM THE PARNAÍBABASIN: THECONTRIBUTIONS OF WILHELM KEGEL. A pioneer in the study of ichnofossils from the Parnaíba Basin, Wilhelm Kegel described materials collected from strata assigned at that time to the Pimenteira, Cabeças, and Longá formations. Since the mid-1960's these specimens remained without proper review and were housed in the paleontological collection of the Departamento Nacional de Produção Mineral in Rio de Janeiro (DNPM/RJ). Because of the remarkable scientific and historical importance of these ichnofossils, their taxonomy and lithostratigraphy have been reviewed in this work, with the following results: (i) the specimens in sample DGM 4940-I, originally described as isolated tubes without ichnogeneric and ichnospecific determination, were classified as Arenicolites isp., possibly originated from the Jaicós Formation; (ii) the specimen in sample DGM 4946-I, cited as straight oriented impressions united by a linear depression, originally attributed to Rusophycus, were identified as Bifungites isp., from the Longá Formation; (iii) the specimen DGM 4945-I, described as Crossochorda maranhensis Kegel was doubtfully classified as Crossopodia? maranhensis (Kegel), probably from the Tianguá Formation; (iv) the specimens DGM 4434-I and DGM 4435-I, described as Crossopodia estrellada Kegel were identified as Phycosiphon incertum Fischer-Ooster and attributed to the Longá Formation; and (v) the specimen DGM 4944-I, from the Pimenteira Formation, described as Spirophyton sp., has been reclassified as Zoophycos isp. The specimen DGM 4923-I, described as Neoskolithos picosensis Kegel is maintained in Kegel's original classification. The specimen DGM 5424-I, described as a ?Homalonotus trace, is not discussed in this paper because it has not been located in the Museu de Ciências da Terra/DNPM/RJ collections.
\end{abstract}

Key words: Silurian-Devonian, Parnaíba Basin, Tianguá Formation, Jaicós Formation, Pimenteira Formation, Longá Formation.

RESUMO - Pioneiro no estudo dos icnofósseis da bacia do Parnaíba, Wilhelm Kegel descreveu o material coletado em estratos atribuídos, na época, às formações Pimenteira, Cabeças e Longá. Desde meados da década de 1960, estes exemplares permaneceram sem uma reavaliação adequada, tendo sido depositados na coleção paleontológica do Departamento Nacional de Produção Mineral do Rio de Janeiro (DNPM/RJ). Devido à importância científica e histórica dos referidos icnofósseis, o presente trabalho efetuou a atualização das atribuições taxonômicas e litoestratigráficas dos mesmos, com os seguintes resultados: (i) os exemplares da amostra DGM 4940-I, descritos originalmente como tubos isolados sem determinação icnogenérica e icnoespecífica, foram classificados como Arenicolites isp., possivelmente oriundos da Formação Jaicós; (ii) na amostra DGM 4946-I, o exemplar referenciado como impressões orientadas em linha reta e unidas por uma depressão linear, atribuído originalmente a Rusophycus, foi identificado como Bifungites isp., da Formação Longá; (iii) o exemplar DGM 4945-I, descrito como Crossochorda maranhensis Kegel foi duvidosamente referenciado como Crossopodia? maranhensis (Kegel), provavelmente da Formação Tianguá; (iv) os exemplares DGM 4434-I e DGM 4435-I, descritos como Crossopodia estrellada Kegel foram identificados como Phycosiphon incertum Fischer-Ooster atribuídos à Formação Longá, e (v) o exemplar DGM 4944-I, da Formação Pimenteira, descrito como Spirophyton sp., foi reclassificado como Zoophycos isp. O exemplar DGM 4923-I, descrito como Neoskolithos picosensis Kegel foi mantido de acordo com sua classificação original. O exemplar descrito como rasto de ?Homalonotus não foi discutido neste trabalho pelo fato do exemplar de número DGM 5424-I não ter sido localizado nas coleções do Museu de Ciências da Terra/DNPM/RJ.

Palavras-chave: Siluriano-Devoniano, bacia do Parnaíba, Formação Tianguá, Formação Jaicós, Formação Pimenteira, Formação Longá.

\section{INTRODUÇÃO}

Os primeiros icnofósseis silurianos e devonianos descritos para a bacia do Parnaíba pelo geólogo Wilhelm Kegel, no período de 1953 a 1966, foram atribuídos às formações Pimenteira, Cabeças e Longá (Kegel, 1953, 1957, 1961, 1965a,b, 1966).
Estudos subsequentes sobre os icnofósseis dessas formações foram efetuados por diversos autores (Campanha, 1974; Carozzi et al., 1975; Brito, 1977; Muniz, 1979, 1981a,b, 1982; Assis \& Fernandes, 1980; Ferreira \& Fernandes, 1983; Fonseca \& Melo, 1987; Carvalho, 1995; Agostinho \& Valença, 1999; Borghi et al., 1999; Silva, 1999; Agostinho \& Fernandes, 2001; Agostinho 
et al., 2001, 2003a,b, 2004; Rodrigues, 2001; Fernandes et al., 2002, 2003; Young et al., 2002; Campelo \& Viana, 2003; Nascimento et al., 2003; Young \& Borghi, 2003; Fernandes \& Fonseca, 2005). Porém, tais estudos utilizaram amostras procedentes de localidades icnofossilíferas diferentes daquelas referidas por Wilhelm Kegel, cujo material permaneceu sem uma reavaliação adequada.

Os trabalhos de Wilhelm Kegel (1890-1971) sobre a geologia e paleontologia da bacia do Parnaíba foram fundamentais para a definição do posicionamento atual das unidades litoestratigráficas desta bacia, especialmente as silurianas e devonianas. Ao corrigir as contradições dos autores anteriores, Kegel constituiu uma coluna estratigráfica bem fundamentada, integrando os dados geológicos com a datação e as inferências paleoambientais obtidas a partir dos fósseis. Nascido na Alemanha, Kegel emigrou para o Brasil após a II Guerra Mundial, em 1949. Geólogo de experiência reconhecida em sua terra natal, no Brasil atuou no Departamento Nacional de Produção Mineral do Rio de Janeiro (DNPM/RJ) e na Escola de Geologia do Rio de Janeiro (Barroso et al., 2008). Os estudos de Kegel se concentraram principalmente no sudeste do Piauí, compreendendo os arredores das cidades de Picos, Oeiras e Valença do Piaú, em área de importância histórica (Santos \& Carvalho, 2009).

Considerando sua relevância científica e histórica, o material icnológico descrito por Kegel (Kegel, 1953, 1957, 1961, 1965a,b, 1966) foi aqui revisado, visando a atualização das atribuições icnotaxonômicas e litoestratigráficas dos icnofósseis previamente estudados.

\section{MATERIAL E MÉTODOS}

O material aqui revisado pertence à coleção do Museu de Ciências da Terra (MCT), no Rio de Janeiro, que hoje abriga a coleção paleontológica da antiga Divisão de Geologia e Mineralogia do Departamento Nacional de Produção Mineral (DNPM). Para tanto, procedeu-se a atualização de suas atribuições taxonômicas e litoestratigráficas (Tabela 1), aprofundando a revisão preliminar realizada por Rodrigues (2001). Para ilustrar os materiais revisados, foram utilizadas imagens originalmente publicadas em Fernandes et al. (2002).

\section{CONTEXTO GEOLÓGICO}

A bacia do Parnaíba abrange uma área de aproximadamente $6.10^{5} \mathrm{~km}^{2}$, cobrindo parte dos estados do Piauí, Maranhão, Tocantins, Pará, Ceará e Bahia. A proposta estratigráfica de Vaz et al. (2007) divide a coluna sedimentar da bacia do Parnaíba em cinco supersequências deposicionais, datadas do Siluriano ao Cretáceo. As unidades litoestratigráficas enfocadas no presente trabalho abrangem do Eosiluriano ao Eocarbonífero, sendo representadas pelas formações superiores do Grupo Serra Grande (formações Ipu, Tianguá e Jaicós - supersequência siluriana) e pelas formações intermediárias do Grupo Canindé (formações Itaim, Pimenteira, Cabeças, Longá e Poti - supersequência mesodevoniana-eocarbonífera).

A Formação Tianguá consiste em folhelhos cinza-escuros (bioturbados, sideríticos e carbonáticos) e arenitos cinzaclaros (finos a médios e feldspáticos), com intercalações de siltitos e folhelhos bioturbados e micáceos, depositados em paleoambiente marinho raso (Góes \& Feijó, 1994). A Formação Jaicós é composta por arenitos cinza médios a grossos, contendo seixos angulares a subangulares, mal selecionados e friáveis, maciços, com estratificação cruzada ou lenticular e, eventualmente, pelitos (Caputo, 1984). O paleoambiente é considerado fluvial entrelaçado (Góes \& Feijó, 1994; Vaz et al., 2007).

A Formação Pimenteira caracteriza-se pela intercalação de arenitos finos, pouco micáceos, com estratificação cruzada hummocky e folhelhos siltosos a argilosos bioturbados, cinzas a pretos, representando a ingressão marinha mais importante da bacia (Melo, 1985). A sedimentação ocorreu em paleoambiente de plataforma rasa dominada por tempestades (Della Fávera, 1990). A Formação Cabeças é composta principalmente por arenitos quartzosos, bem selecionados, de granulometria muito fina a grossa e coloração esbranquiçada a arroxeada, com intercalações de siltitos, arenitos conglomeráticos e raramente folhelhos. A estratificação cruzada assintótica predomina nesta unidade, ocorrendo intercalada a arenitos com estratificação cruzada hummocky em sua base, no

Tabela 1. Resumo da litologia, ambiente e idade das unidades litoestratigráficas da bacia do Parnaíba (a partir de Góes \& Feijó, 1994 e Vaz et al., 2007) com a distribuição dos icnofósseis estudados por Kegel $(1953,1957,1961,1965 a, b, 1966)$ e enfocados no presente trabalho.

Table 1. Summary of the lithology, environment, and age of lithostratigraphic units of the Parnaíba Basin (from Góes \& Feijó, 1994 and Vaz et al., 2007), showing the distribution of trace fossils studied by Kegel $(1953,1957,1961,1965 a, b, 1966)$ and focused in this work.

\begin{tabular}{|c|c|c|c|c|}
\hline $\begin{array}{l}\text { Unidade } \\
\text { litoestratigráfica }\end{array}$ & Litologia & Ambiente & Idade & Icnofósseis \\
\hline Formação Longá & $\begin{array}{l}\text { Folhelhos cinza-escuros muito laminados, } \\
\text { intercalados com arenitos e siltitos } \\
\text { cinza-claros a esbranquiçados }\end{array}$ & $\begin{array}{l}\text { Marinho-plataformal } \\
\text { raso }\end{array}$ & $\begin{array}{l}\text { Tournaisiano } \\
\text { - } \\
\text { Fameniano }\end{array}$ & $\begin{array}{l}\text { Phycosiphon incertum; } \\
\text { Bifungites isp. }\end{array}$ \\
\hline Formação Pimenteira & $\begin{array}{c}\text { Arenitos finos com estratificação cruzada } \\
\text { hummocky e folhelhos siltosos a argilosos, } \\
\text { cinzas a pretos }\end{array}$ & $\begin{array}{l}\text { Marinho-plataformal } \\
\text { raso }\end{array}$ & $\begin{array}{l}\text { eofameniano } \\
\text { - } \\
\text { neo-eifeliano }\end{array}$ & $\begin{array}{l}\text { Neoskolithos } \\
\text { picosensis; Zoophycos } \\
\text { isp. }\end{array}$ \\
\hline Formação Jaicós & $\begin{array}{l}\text { Arenitos cinzas, médios a grossos, com } \\
\text { estratificação cruzada e eventualmente } \\
\text { intercalação de pelitos }\end{array}$ & Fluvial entrelaçado & $\begin{array}{c}\text { Praguiano } \\
\text { - } \\
\text { Landoveriano }\end{array}$ & Arenicolites isp. \\
\hline Formação Tianguá & $\begin{array}{l}\text { Arenitos cinza-claros (finos a médios) e } \\
\text { folhelhos cinza-escuros }\end{array}$ & $\begin{array}{l}\text { Marinho-plataformal } \\
\text { raso }\end{array}$ & Landoveriano & $\begin{array}{l}\text { Crossopodia? } \\
\text { maranhensis }\end{array}$ \\
\hline
\end{tabular}


Membro Passagem. Na porção superior deste membro, os arenitos seriam mais semelhantes aos do Membro Oeiras, de granulometria fina a média e com pouca mica (Caputo, 1984). Rumo ao topo, passam a predominar arenitos fluidizados, associados a tilitos/diamictitos, ritmitos e pavimentos estriados, especialmente na borda oeste e subsuperfície da bacia (Kegel, 1953; Caputo et al., 2005). O paleoambiente inferido seria flúvio-deltaico distal a plataformal raso, sob a influência de inundações episódicas e tempestades (Ponciano \& Della Fávera, 2009). A Formação Longá apresenta principalmente folhelhos cinza-escuros a pretos, em parte arroxeados, homogêneos ou bem laminados e bioturbados. Em sua porção média são encontrados arenitos e siltitos laminados cinza-claros a esbranquiçados (Vaz et al., 2007). Segundo Góes \& Feijó (1994), o paleoambiente seria marinho raso dominado por tempestades.

\section{PROCEDÊNCIA DAS AMOSTRAS}

Kegel (1953, 1957, 1961, 1965a,b, 1966) descreveu os icnofósseis procedentes de nove localidades da bacia do Parnaíba, sendo duas ocorrências em poços da Petrobras no estado do Maranhão e sete registros em afloramentos situados no estado do Piauí (Figura 1). Entretanto, de acordo com José Henrique Gonçalves de Melo (com. pess., 2011), os registros de Kegel (1966) para os poços da Petrobras apresentam alguns problemas de interpretação. O poço VG1 citado por Kegel (1966) como furo Riachão, efetuado nas proximidades da cidade homônima, no sudoeste do estado do Maranhão (Figura 1, localidade 1), corresponde ao poço 1-VG-1MA. Este poço, devido a problemas mecânicos durante sua perfuração, foi abandonado a 1.029,4 $\mathrm{m}$ de profundidade, continuando através do poço 1-VG-1R-MA, perfurado ao lado do primeiro. O autor, ao referir-se ao testemunho coletado,

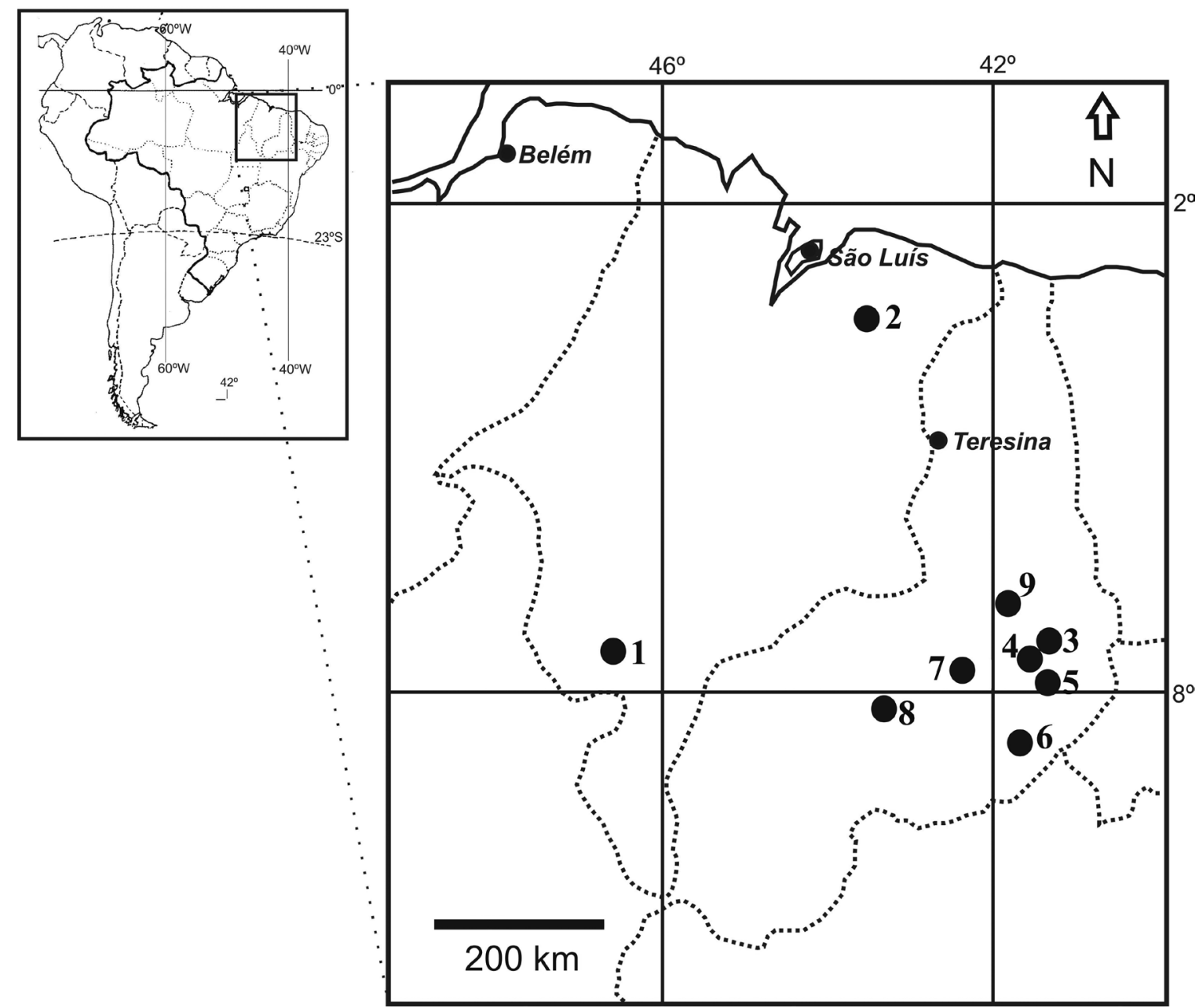

Figura 1. Mapa de localização das ocorrências descritas por Kegel para a bacia do Parnaíba, nos estados do Maranhão e Piauí: (1) poço 1-VG1R-MA, nas proximidades da cidade de Riachão (MA); (2) poço 2-VG-1-MA, nas proximidades de Vargem Grande (MA); (3) Morro de Ipoeira, nas cercanias de Picos (PI); (4) km 309 da BR-316 (PI); (5) BR-020, ao sul do entroncamento das estradas Picos-Jaicós/Picos-Itainópolis (PI); (6) BR-020, ao sul de São João do Piauí (PI); (7) PI-143, ao sul de Santo Inácio do Piauí (PI); (8) BR-324, próximo à cidade de Canto do Buriti (PI); e (9) $8 \mathrm{~km}$ a oeste da cidade de Elesbão Veloso (PI).

Figure 1. Location map of the trace fossil occurrences described by Kegel from the Parnaíba Basin, in the States of Maranhão e Piauí: (1) well 1-VG1R-MA, in the vicinity of Riachão (MA); (2) well 2-VG-1-MA, near Vargem Grande (MA); (3) Morro de Ipoeira, near Picos (PI); (4) km 309 of BR-316 (PI); (5) BR-020, to the south of the junction with the Picos-Jaicós/Picos-Itainópolis roads (PI); (6) BR-020, south of São João do Piauí (PI); (7) PI143 , south of Santo Inácio do Piauí (PI); (8) BR-324, in the vicinity of Canto do Buriti (PI); and (9) outcrop situated 8 km west of Elesbão Veloso (PI). 
mencionou-o como procedente da profundidade de 1.530 m-1.535 m, o que não é compatível com a profundidade atingida pelo poço 1-VG-1-MA. Fica, portanto, a dúvida se o referido testemunho não seria do poço vizinho. A julgar pelos testemunhos do poço 1-VG-1R-MA, na referida profundidade ele se encontraria dentro da Formação Jaicós, que abrange do Eosiluriano ao Eodevoniano (Landoveriano-Praguiano), contrariando a atribuição de Kegel (1966) para a Formação Cabeças (mesodevoniana a neodevoniana).

$\mathrm{O}$ outro poço com ocorrência de icnofósseis, citado por Kegel (1966) como VGst-1-MA, corresponde ao poço 2-VG-1MA, perfurado nas proximidades da cidade de Vargem Grande, nordeste do Maranhão (Figura 1, localidade 2). A profundidade de 1.379 m-1.380 m, neste testemunho, corresponde a depósitos da Formação Tianguá, de idade eossiluriana (Landoveriano) (Grahn et al., 2005), não sendo compatível com a Formação Longá, conforme as observações de Kegel (1966).

Os sete registros assinalados em afloramentos, todos no Piauí, foram atribuídos às formações Pimenteira e Longá. Cinco destes seriam referentes à Formação Pimenteira: (i) Morro de Ipoeira, situado a alguns quilômetros de Picos. Provavelmente é o atual morro da rádio de Picos, quase totalmente ocupado por casas e comércios (Figura 1, localidade 3); (ii) afloramento situado na margem da rodovia Picos-Recife a $9 \mathrm{~km}$ da cidade de Picos, no atual km $309 \mathrm{da}$ BR-316 (entre os antigos marcos 115 e 116 da BR 24, na época das coletas de Kegel) (Figura 1, localidade 4); (iii) afloramento da BR-020 (Picos-Itainópolis) 24 km após Picos, ao sul do entroncamento das estradas Picos-Jaicós (atual BR-407)/Picos-Itainópolis (antiga Picos-Jenipapo) (Figura 1, localidade 5); (iv) afloramento situado alguns quilômetros ao sul de São João do Piauí, na atual BR-020, em direção à São Raimundo Nonato (representa o "banco branco" de Kegel, base da Formação Pimenteira) (Figura 1, localidade 6); (v) afloramento situado na estrada Oeiras-Simplício Mendes (atual PI-143), alguns quilômetros ao sul de Santo Inácio do Piauí (antigo Brejo de Santo Inácio) (Figura 1, localidade 7).
Os outros dois afloramentos são atribuídos à Formação Longá: (i) afloramento próximo à cidade de Canto do Buriti, na BR324 (PI-140) que liga esta cidade a São Raimundo Nonato (Figura 1, localidade 8); e (ii) afloramento situado $8 \mathrm{~km}$ a oeste da cidade de Elesbão Veloso (Figura 1, localidade 9).

\section{ICNOTAXONOMIA}

Icnogênero Arenicolites Salter, 1957

Arenicolites isp.

(Figuras 2A-B)

1966 Rastos. Kegel, p. 23, fig. 3, foto 8. 2001 Arenicolites isp. Rodrigues, p. 124.

2002 Arenicolites isp. Fernandes et al., p. 15, figs. 5-6.

Material. DGM 4940-I.

Descrição. Escavações em forma de tubos em U, sem spreiten, dispostas verticalmente e sem intercruzamento (Figura 2A). Comprimento total do espécime melhor preservado igual a $82,88 \mathrm{~mm}$; diâmetro das escavações variável, de 7,02 mm a $15,21 \mathrm{~mm}$. Os tubos verticais observados no corte em planta do testemunho encontram-se em aparente pareamento, confirmando a forma em U (Figura 2B); ocorrem também indícios de outras escavações de maior comprimento.

Discussão. Kegel (1966) mencionou pela primeira vez essas escavações como se fossem tubos isolados, interpretando-os como semelhantes a Cylindricum, Laevicyclus e Planolites. As características citadas por Häntzschel (1975) para esses icnogêneros, contudo, não são observadas no exemplar analisado. Cylindricum se caracteriza por escavações preservadas em grupos, em forma de tubos de ensaio, com arredondamento na parte inferior, paredes lisas e vários centímetros de comprimento, orientadas perpendicularmente ao plano de estratificação. O icnofóssil do testemunho analisado possui tubos pareados em forma de $U$ e não
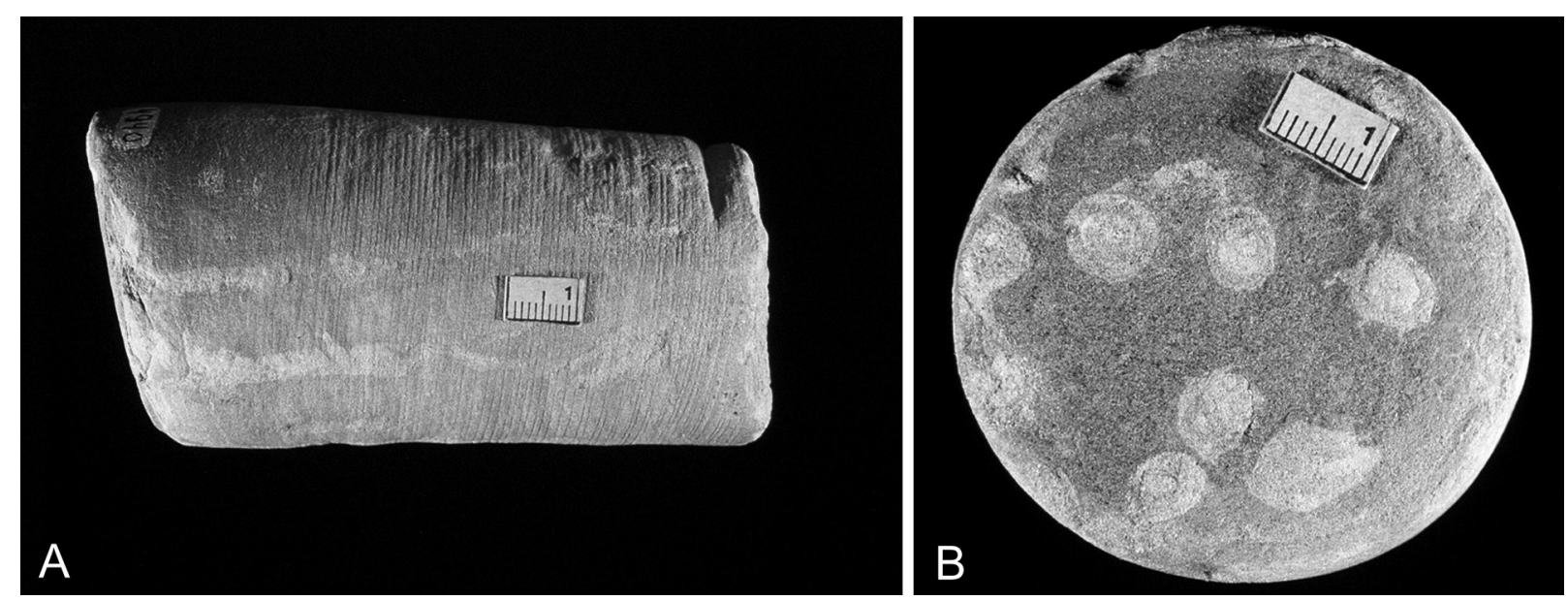

Figura 2. Arenicolites isp. (testemunho do poço 1-VG-1R-MA, DGM 4940-I). A, vista transversal mostrando escavação em forma de U, sem spreiten, disposta verticalmente; B, corte em planta mostrando os tubos em seção horizontal, dispostos em aparente pareamento, típico de Arenicolites. Escalas $=1 \mathrm{~cm}$.

Figure 2. Arenicolites isp. (core from 1-VG-1R-MA, DGM 4940-I). A, cross section view showing U-shaped vertical excavation lacking spreiten; B, plan view showing apparently paired tubes in horizontal section, typical of Arenicolites. Scale bars $=1 \mathrm{~cm}$. 
isolados, como corresponde à Cylindricum. Laevicyclus caracteriza-se por corpos aproximadamente cilíndricos dispostos verticalmente ao plano de estratificação, contendo um canal central, visível como círculos concêntricos regulares com diâmetro de vários centímetros; tais características não são encontradas no exemplar em estudo, pois este não possui um canal central, sendo ausente qualquer estrutura interna no seu preenchimento. Planolites caracteriza-se por escavações cilíndricas ou subcilíndricas preenchidas, retas a levemente curvas, não ramificadas, geralmente de disposição mais ou menos horizontal ou oblíqua ao plano de estratificação, penetrando o sedimento em direção e curso irregular, podendo intercruzar-se. Essas características também são irrelevantes para as escavações estudadas, pois as mesmas são retas, dispostas verticalmente ao plano de estratificação e sem intercruzamentos. Portanto, concluiu-se que a comparação e interpretação destas estruturas feita por Kegel (1966) como semelhantes a tais icnogêneros não foi adequada, visto que as características apresentadas pelo exemplar são correspondentes a Arenicolites. Apesar de ser comum em ambientes marinhos de águas rasas, Arenicolites já foi registrado em ambientes de águas profundas e em ambientes não-marinhos (e.g. Fillion \& Pickerill, 1990; Bromley, 1996; Buatois \& Mángano, 2004; Netto, 2007). Nos ambientes não marinhos, ocorre nas icnofácies Scoyenia (eólicos interdunas) e Mermia (depósitos de água doce) (e.g. Buatois \& Mángano, 1996, 2004). Distribui-se do Cambriano Inferior ao Holoceno (Fillion \& Pickerill, 1990). Apesar de sua ocorrência ter sido atribuída por Kegel (1966) à Formação Cabeças, o material revisado é reinterpretado como pertencente à Formação Jaicós.

Procedência. Provável testemunho do poço 1-VG-1R-MA da Petrobras, com profundidade de 1.530 m-1.535 m (segundo Kegel, 1966), nas proximidades da cidade de Riachão, sudoeste do Maranhão.

Unidade litoestratigráfica. Possivelmente pertencente à Formação Jaicós, do Grupo Serra Grande, e não à Formação Cabeças, segundo a interpretação original de Kegel (1966).
Icnogênero Bifungites Desio, 1940

Bifungites isp.

(Figuras 3A-B)

1965 Rastos. Kegel, p.6, figs. 1, 2.

1965 Rusophycus isp. Kegel, p.8, figs. 1, 2.

2001 Bifungites isp. Rodrigues, p. 124.

Material. DGM 4946-I.

Descrição. A análise da amostra DGM 4946-I revelou a existência de dois exemplares de Bifungites. O primeiro, correspondente a um exemplar incompleto (Figura 3A), encontra-se representado somente pelo corpo terminal preservado em epirrelevo convexo, de formato oval, com comprimento igual a $10,65 \mathrm{~mm}$ e largura igual a $6,85 \mathrm{~mm}$, no qual do centro para a extremidade segue uma elevação tubular semelhante a uma crista. O segundo está representado por um exemplar completo (Figura 3B) preservado em semirrelevo convexo, cujos corpos terminais apresentam forma de seta e se comunicam por um eixo central subcilíndrico, e possui as seguintes dimensões: eixo central com comprimento de 14,45 $\mathrm{mm}$, extremidades com comprimento variável entre $4,00 \mathrm{~mm}$ e 5,22 mm, largura das bases dos corpos terminais variando de $4,22 \mathrm{~mm}$ a $5,77 \mathrm{~mm}$, e comprimento total do exemplar igual a $23,70 \mathrm{~mm}$.

Discussão. Com relação ao exemplar incompleto (Figura 3A), Kegel (1965b) o descreveu como semelhante a Rusophycus. Rusophycus caracteriza-se como escavações bilobadas intraestratais e epiestratais, produzidas por trilobitas em posição estática face ao substrato (de repouso), com orientação horizontal e cujos lobos podem apresentar-se de forma oval, podendo ser lisas ou ter ranhuras oblíquas (Kegel, 1961). A estrutura descrita por Kegel, entretanto, pelas suas características morfológicas, corresponde a um corpo terminal de Bifungites. Com relação ao exemplar da Figura 3B, Kegel (1965b) mencionou, para a parte superior esquerda da amostra, impressões orientadas em linha reta e
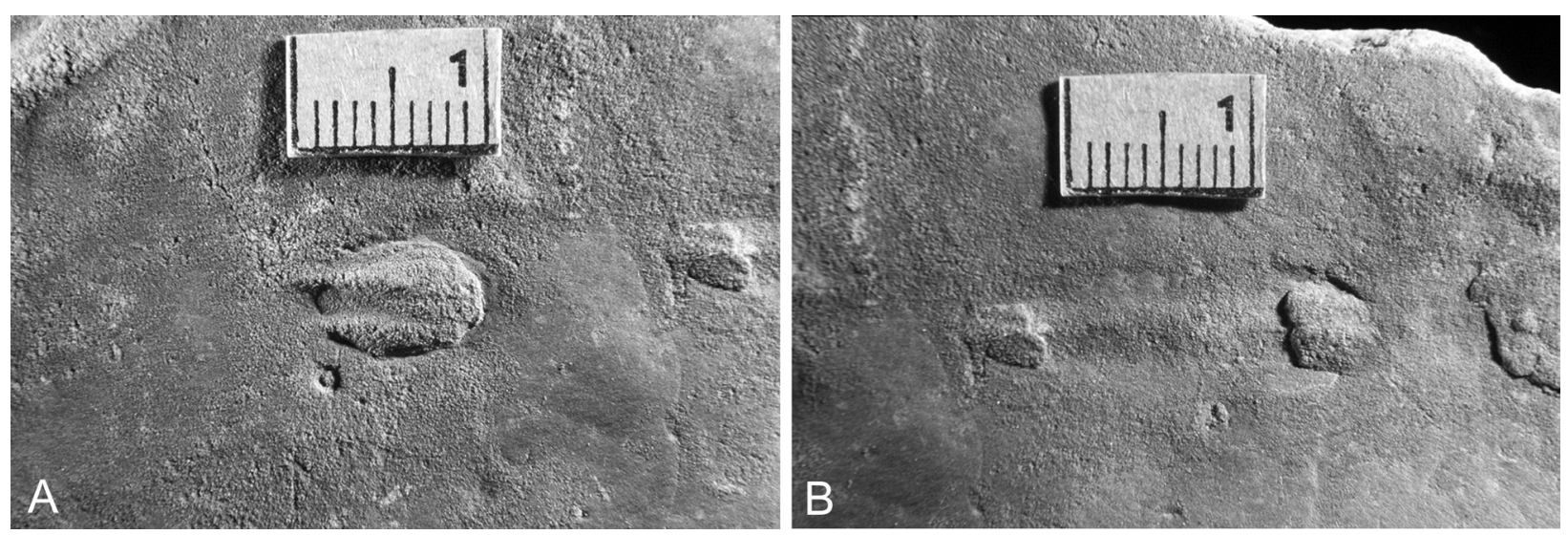

Figura 3. Bifungites isp. (DGM 4946-I). A, exemplar incompleto preservado em semi-relevo convexo, de formato oval, apresentando leve elevação tubular no centro do corpo terminal; B, exemplar completo, preservado em semi-relevo convexo, com dois corpos terminais em forma de seta interligados por um eixo central. Escalas $=1 \mathrm{~cm}$.

Figure 3. Bifungites isp. (DGM 4946-I). A, incomplete, oval-shaped specimen preserved in convex semirelief, showing a slight tubular elevation in the axial shaft; $\mathbf{B}$, complete specimen preserved in convex semirelief, with double arrow-shaped terminations connected by a straight shaft. Scale bars $=1 \mathrm{~cm}$. 
unidas por uma depressão linear. A análise da amostra resultou na identificação dessas impressões como correspondentes aos corpos terminais de um exemplar de Bifungites e, a referida depressão linear, o seu eixo central. Deficiências na preservação dos exemplares não permitiram observações mais detalhadas de suas características morfológicas, levando à identificação de ambos como Bifungites isp.. Bifungites ocorre na icnofácies Cruziana e distribui-se do Pré-Cambriano ao Permiano (Turner \& Benton, 1983).

Procedência. Afloramento situado próximo à cidade de Canto do Buriti, sul do Piauí, na BR-324 (PI-140) que liga esta cidade a São Raimundo Nonato (antigo km 68,9, na época da coleta do material em 1962).

Unidade litoestratigráfica. Atribuída à Formação Cabeças por Kegel (1965b); porém, devido à localização geográfica, a amostra deve ser oriunda da Formação Longá.

$$
\text { Icnogênero Crossopodia M'Coy, } 1851
$$

\section{Crossopodia? maranhensis (Kegel, 1966)}

(Figuras 4A-B)

1966 Crossochorda maranhensis. Kegel, p. 31, fig. 4, fotos 10, 11. 2001 Crossopodia? maranhensis. Rodrigues, p. 124.

2002 Crossopodia maranhensis. Fernandes et al., p. 41-42, figs. 37-38.

Material. DGM 4945-I (holótipo).

Descrição. Pista bilobada meandrante, lisa, preservada em epirrelevo côncavo, dotada de uma crista mediana, com comprimento total de $83,20 \mathrm{~mm}$ e largura máxima de 3,0 $\mathrm{mm}$. Ocorre também em hiporrelevo convexo correspondente. Discussão. Kegel (1966) descreveu esta pista como sendo uma icnoespécie nova de Crossochorda Schimper, 1879, icnogênero atualmente considerado sinônimo de Crossopodia M'Coy, 1851. Entretanto, a atribuição de Crossochorda maranhensis à Crossopodia é problemática, pois este icnogênero apresenta como uma de suas características uma franja densa e larga do lado de cada lobo ou sulco, a qual não é observada no material estudado. Por esta razão, o citado exemplar encontra-se duvidosamente atribuído à Crossopodia. Este icnogênero ocorre na icnofácies Cruziana, com uma distribuição do Cambriano Inferior ao Carbonífero (Fernandes et al., 2002). Outro icnogênero com características morfológicas semelhantes às descritas para o exemplar analisado é Didymaulichnus Young, 1972; porém, as escavações bilobadas de Didymaulichnus contêm cerca de $2 \mathrm{~cm}$ de largura, dimensão superior ao exemplar em questão. Procedência. Testemunho do poço 2-VG-1-MA da Petrobras, de profundidade $1.379 \mathrm{~m}-1.380 \mathrm{~m}$, nas proximidades da cidade de Vargem Grande, nordeste do Maranhão.

Unidade litoestratigráfica. Formação Tianguá (Grupo Serra Grande), de idade eossiluriana (Landoveriano), atualizando o posicionamento de Kegel (1966), que considerava a amostra como oriunda da Formação Longá ou Cabeças.

Icnogênero Neoskolithos Kegel, 1966

Neoskolithos picosensis Kegel, 1966

(Figuras 5A-B)

1966 Neoskolithos picosensis. Kegel, p. 19-23, fig. 2, fotos 6-7. 2002 Neoskolithos picosensis. Fernandes et al., p. 107-108, figs. 88-90.

Material. DGM 4923-I (holótipo).

Descrição. Agrupamento de escavações dispostas perpendicularmente à estratificação ou levemente inclinados, individualizados e com finos interstícios entre si. As escavações apresentam base arredondada, por vezes aparentemente plana, principalmente nos casos onde se verifica uma possível geminação entre elas. A forma e o diâmetro das escavações são irregulares, sendo pouco maior na sua base, e com o sedimento que preenche os interstícios mascarando em grande parte a sua morfologia. Porém, nem sempre as escavações apresentam uma seção transversal circular, mostrando-se então achatadas lateralmente. Medidas observadas: diâmetro entre 2,94 mm e 9,30 $\mathrm{mm}$, mas as formas aparentemente geminadas na
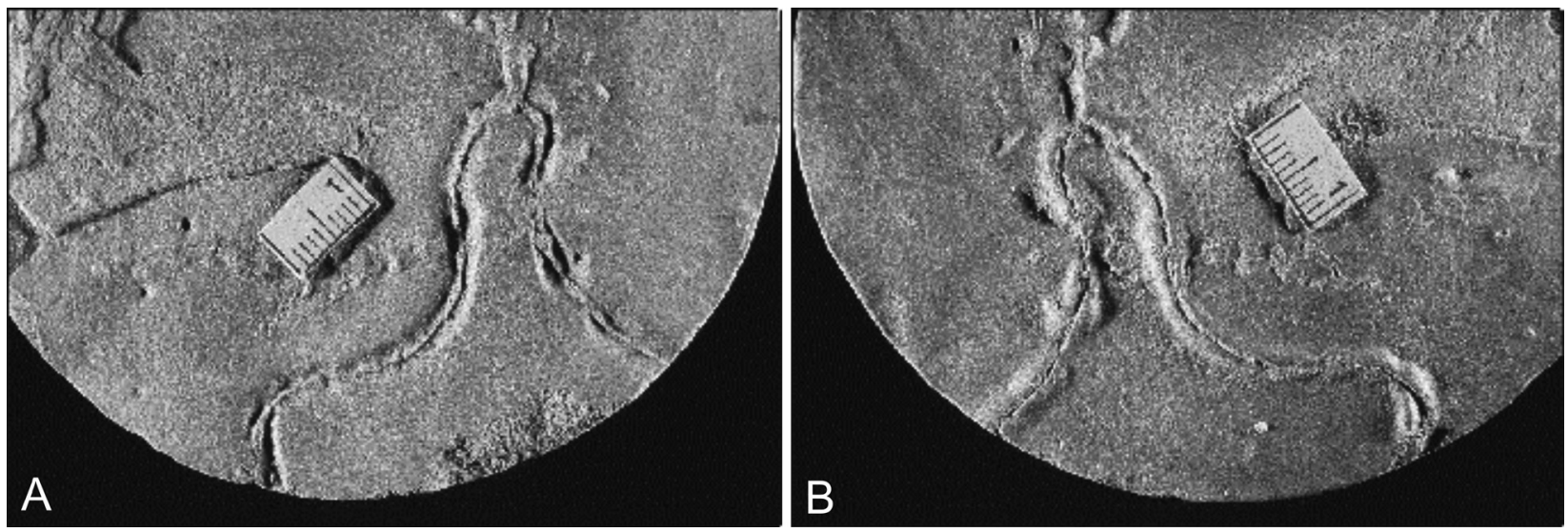

Figura 4. Crossopodia? maranhensis (Kegel, 1966) (DGM 4945-I, holótipo). A, pista bilobada meandrante, lisa, preservada em epirrelevo côncavo e hiporrelevo convexo; B, crista mediana bem definida. Escalas $=1 \mathrm{~cm}$.

Figure 4. Crossopodia? maranhensis (Kegel, 1966) (DGM 4945-I, holotype). A, smooth, meandering, bilobed trail preserved in concave epirelief and convex hiporelief; $\mathbf{B}$, well-defined median ridge. Scale bars $=1 \mathrm{~cm}$. 


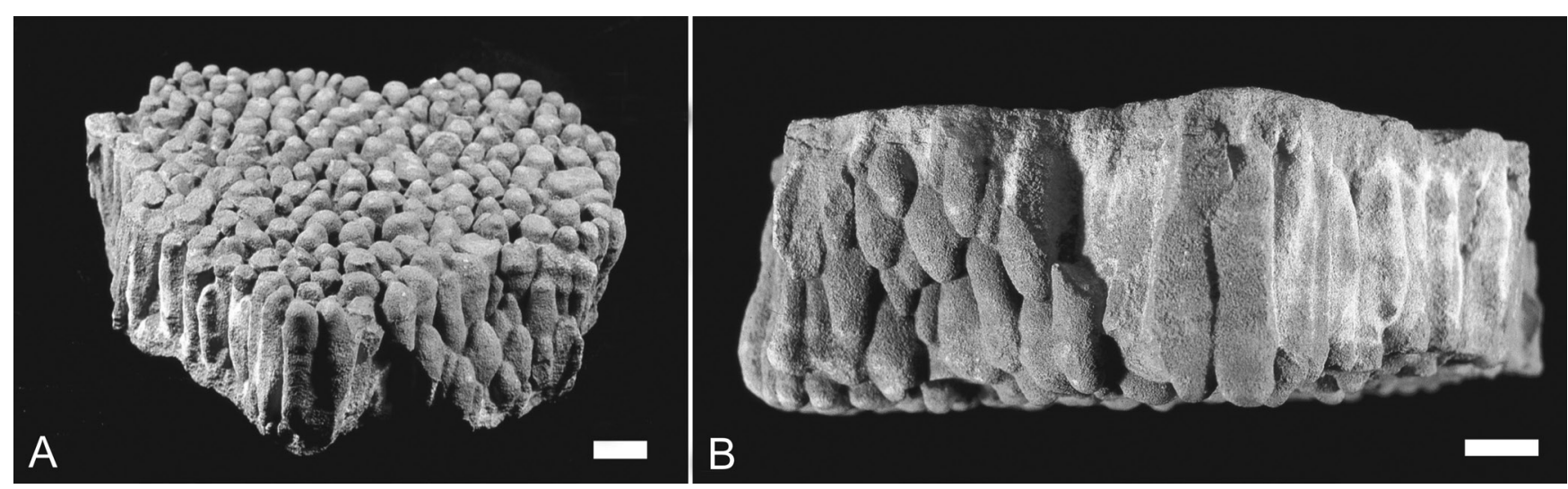

Figura 5. Neoskolithos picosensis Kegel, 1966 (DGM 4923-I, holótipo). A, vista geral inferior onde se encontram ressaltadas as bases de contorno irregular arredondado dos tubos; B, vista lateral demonstrando a irregularidade no diâmetro e no comprimento dos tubos, aparentemente unidos devido ao preenchimento dos interstícios. Escalas $=1 \mathrm{~cm}$.

Figure 5. Neoskolithos picosensis Kegel, 1966 (DGM 4923-I, holotype). A, lower overview highlighting the rounded, irregular basal contour of the tubes; $\mathbf{B}$, lateral view showing irregularity in diameter and length of the tubes, apparently united due to the infilling of interstices. Scale bars $=1 \mathrm{~cm}$.

base podem atingir entre $9,23 \mathrm{~mm}$ e $13,51 \mathrm{~mm}$ de diâmetro; comprimento de $6,23 \mathrm{~mm}$ a $33,92 \mathrm{~mm}$; interstícios entre as escavações, medidos entre as bases arredondadas, variam entre $0,52 \mathrm{~mm}$ e $2,99 \mathrm{~mm}$.

Discussão. Neoskolithos é um icnogênero monoespecífico, até o momento identificado somente nos sedimentos devonianos da Formação Pimenteira. Difere do tradicional icnogênero Skolithos por suas dimensões menores e uma forma mais irregular (Häntzschel, 1975). Anteriormente registrado apenas na região de Picos, também já foi encontrado no topo do afloramento da entrada da cidade de Itainópolis (base da Formação Pimenteira). Ainda não são conhecidas amostras de testemunhos com Neoskolithos, mas o mesmo poderia ser identificado nas seções longitudinais como cortes de agrupamentos de escavações verticais curtas afunilando-se levemente em direção à base, arredondada e achatada (Fernandes et al., 2002). Neoskolithos também já foi interpretado como estruturas inorgânicas por Alpert (1974), sendo por ele considerado como nomen nudum, embora tenha sido validado por Häntzschel (1975), motivo pelo qual foi mantido no presente artigo.

Procedência. Morro de Ipoeira, situado a alguns quilômetros de Picos, estado do Piauí. Provavelmente é o atual morro da rádio de Picos, quase totalmente ocupado por casas e restaurantes. Unidade litoestratigráfica. Atribuída à Formação Pimenteira por Kegel (1966), posicionamento confirmado pelas observações atuais.

Icnogênero Phycosiphon Fischer-Ooster, 1858

Phycosiphon incertum Fischer-Ooster, 1858

(Figuras 6A-E)

1957 Myrianites sp. Kegel, p. 9, figs. 6-8.

1966 Nereites sp. Kegel, p. 8-12, fotos 1-4.

1966 Crossopodia estrellada. Kegel, p. 14, fig. A, foto 5.

2001 Phycosiphon estrellada. Rodrigues, p. 124.

2002 Nereites isp. Fernandes et al., p. 109-110, figs. 92-94. 2002 Phycosiphon estrellada. Fernandes et al., p. 123, figs. 106-107.
Material. DGM 4434-I, 4435-I, 4939-I, 4924-I, 4925-I, 4926-I e 4927-I.

Descrição. Escavações assemelhadas a pequenos lobos em forma de laços em $\mathrm{U}$, com poucas ramificações e ocorrendo em grande número, formando um sistema complexo. Lobos medindo de 4,25 mm a 8,20 $\mathrm{mm}$ de comprimento, com largura total variável entre $2,30 \mathrm{~mm}$ e $4,28 \mathrm{~mm}$, e espessura dos tubos aproximadamente de 0,97 $\mathrm{mm}$ a 1,80 mm. Apesar de a presença de spreiten ser comum em Phycosiphon, a estrutura não foi observada nos espécimes revisados.

Discussão. Kegel (1957) descreveu originalmente esses exemplares como Myrianites (= Nereites MacLeay, 1839, segundo Häntzschel, 1975). Posteriormente, Kegel (1966) redescreveu os exemplares como Crossopodia estrellada por considerar que, apesar da morfologia e da existência de meandros lembrarem morfologias identificadas como Myrianites, os exemplares estudados se distinguiam pela ausência de tripartição longitudinal característica de Myrianites. Entretanto, a morfologia das escavações não se enquadra na diagnose do icnogênero Crossopodia, representado por escavações meandrantes, curvas ou retas, com uma franja densa e larga de cada lado, possuindo na sua maioria uma crista mediana, características não observadas nos exemplares estudados. Os exemplares apresentam as características do icnogênero Phycosiphon, cuja escavação é diagnosticada como uma estrutura pequena, apresentando spreiten em lobos proeminentes formado a partir de um retropreenchimento zonado composto por um núcleo escuro e um envoltório claro. Localmente, o spreiten pode ser interrompido, preservando-se apenas um único tubo com retropreenchimento, segundo Wetzel \& Bromley (1994). A composição arenítica da rocha matriz não permitiu, entretanto, a preservação dos spreiten. Phycosiphon é representado por uma única icnoespécie, cujas características não se diferenciam das apresentadas pelos exemplares descritos por Kegel (1957), razão pela qual Crossopodia estrellada é colocada aqui em sinonímia com Phycosiphon incertum Fischer-Ooster, 1858. Apesar de relacionado à icnofácies Zoophycos, Phycosiphon também pode ocorrer 

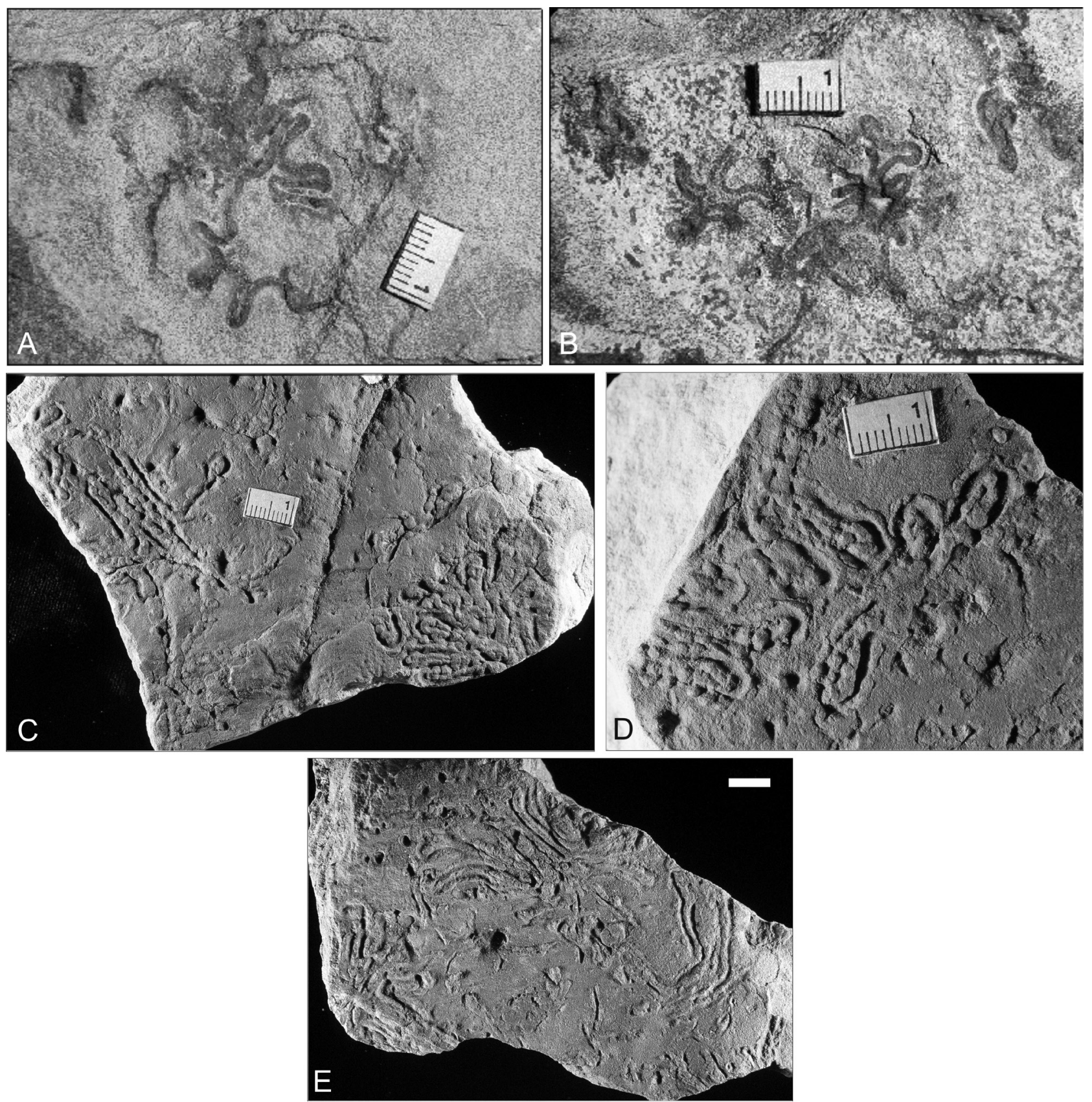

Figura 6. Phycosiphon incertum Fischer-Ooster, 1858: A, DGM 4434-I; B, DGM 4435-I; C, DGM 4924-I; D, DGM 4926-I; E, DGM 4925-I. Escalas $=1 \mathrm{~cm}$.

Figure 6. Phycosiphon incertum Fischer-Ooster, 1858. Scale bars $=1 \mathrm{~cm}$.

em outras icnofácies, tendo sido registrado em sedimentos síltico-argilosos e arenosos presentes em ambientes marinhos rasos a profundos (Wetzel \& Bromley, 1994). Distribui-se do Ordoviciano ao Holoceno.

Procedência. Os exemplares DGM 4434-I, 4435-I e 4939-I são oriundos do afloramento situado $8 \mathrm{~km}$ a oeste da cidade de Elesbão Veloso, representando a Formação Longá, segundo Kegel (1957) e o presente estudo. Já as amostras 4926-I e 4927-I foram coletadas num afloramento da BR-020 (Picos-Itainópolis) 24 km após Picos, ao sul do entroncamento das estradas Picos-Jaicós (atual BR-407)/ Picos-Itainópolis (antiga Picos-Jenipapo), na margem sudeste da bacia do Parnaíba, sendo realmente procedentes da Formação Pimenteira. Apesar dos exemplares 4924I e 4925-I terem sido tombados como procedentes do afloramento anterior, as diferenças sedimentológicas e o registro de outra localidade em Kegel (1966) indicam que estes devem ser os icnofósseis do "banco branco" de Kegel, base da Formação Pimenteira, situado alguns quilômetros ao sul de São João do Piauí, na atual BR-020, em direção à São Raimundo Nonato.

Unidade litoestratigráfica. Formação Longá para as amostras 4434-I, 4435-I e 4939-I e Formação Pimenteira para os exemplares 4924-I, 4925-I, 4926-I e 4927-I. 


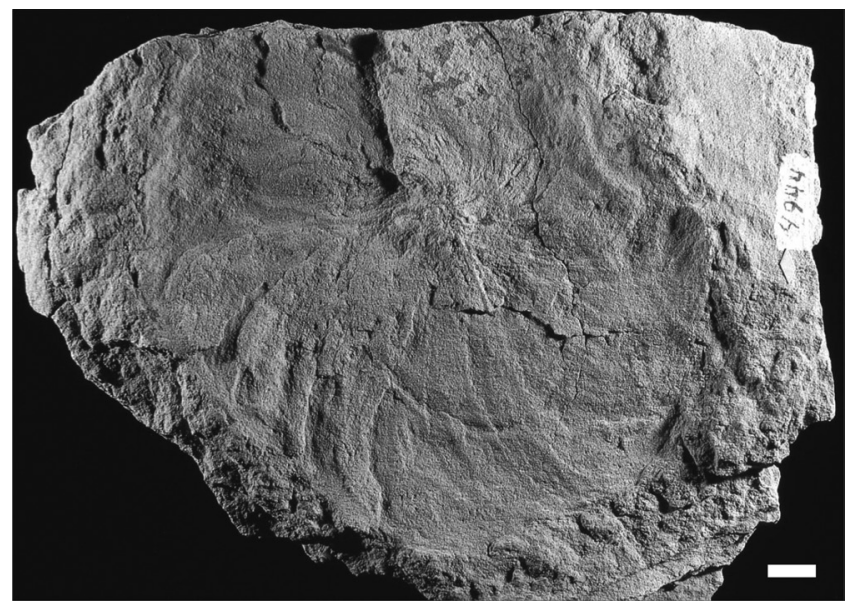

Figura 7. Zoophycos isp.: exemplar DGM 4944-I, preservado em planta. Escala $=1 \mathrm{~cm}$.

Figure 7. Zoophycos isp.: specimen DGM 4944-I, preserved in plan view. Scale bar $=1 \mathrm{~cm}$.

Icnogênero Zoophycos Massalongo, 1855

Zoophycos isp.

(Figura 7)

1966 Spirophyton sp. Kegel, p. 27-29, foto 9.

2002 Spirophyton isp. Fernandes et al., p. 147, fig. 135.

Material. DGM 4944-I.

Descrição. Escavação com $13 \mathrm{~cm}$ a $14 \mathrm{~cm}$ de diâmetro e orientação horizontal a oblíqua, constituída por uma estrutura helicoidal com ligeira elevação central. Encontra-se coberto por linhas muito finas, quase radiais, curvadas no mesmo sentido, do centro à periferia, sendo mais fortes na região em torno da elevação central, sendo a expressão em planta do spreiten.

Discussão. Até a revisão icnotaxonômica realizada por Olivero (2007), Spirophyton e Zoophycos eram descritos como icnogêneros portadores de morfologias bastante semelhantes sendo uma de suas diferenças o tamanho (Häntzschel, 1975): Spirophyton representaria as formas de menor tamanho (com diâmetro em torno de $8 \mathrm{~cm}$ ), com contorno circular das laminae e sem tendências à formação de lobos, enquanto Zoophycos corresponderia às formas de menor altura, mas de maior diâmetro das laminae que, segundo Gerard \& Bromley (2008), poderia chegar até $1 \mathrm{~m}$. Entretanto, ao revisar o icnogênero Zoophycos, Olivero (2007) incluiu Spirophyton como um de seus sinônimos. O exemplar descrito por Kegel (1966) como Spirophyton, portanto, passa a ser identificado como Zoophycos isp. Os prováveis produtores de Zoophycos seriam animais vermiformes viventes em substratos lamosos (Seilacher, 2003) e amplamente adaptados em diversos ambientes, ocorrendo desde a plataforma interna até bacias de grande profundidade (Olivero, 2007). De um modo geral, entretanto, o predomínio de baixas taxas de oxigenação parece ser o principal fator que condiciona o estabelecimento de icnocenoses de Zoophycos, em condições sob as quais poucos animais são capazes de viver. Zoophycos apresenta uma distribuição do Cambriano ao Holoceno (Olivero, 2007).
Procedência. Afloramento situado na estrada OeirasSimplício Mendes (atual PI-143), alguns quilômetros ao sul de Santo Inácio do Piauí (antigo Brejo de Santo Inácio), no Piauí. Unidade litoestratigráfica. Segundo Kegel (1966) e o presente trabalho o afloramento pertence à Formação Pimenteira.

\section{CONSIDERAÇÕES FINAIS}

Após a atualização das unidades estratigráficas onde foram coletados os icnofósseis analisados, constata-se que nenhum dos exemplares estudados por Kegel (1965b, 1966) pertence à Formação Cabeças, estando representados apenas nas formações Tianguá, Jaicós, Pimenteira e Longá.

A revisão taxonômica e litoestratigráfica dos icnofósseis descritos originalmente por Kegel resultou nas seguintes alterações: (i) a partir da nova análise do exemplar DGM 4940-I, concluiu-se que as comparações e interpretações feitas por Kegel (1966) relacionadas aos icnogêneros Cylindricum, Laevicyclus e Planolites não foram adequadas, já que as suas características correspondem às do icnogênero Arenicolites, sendo os exemplares da amostra classificados como Arenicolites isp.; (ii) o exemplar DGM 4946-I, identificado por Kegel (1965) como uma forma de Rusophycus, corresponde a um corpo terminal de um exemplar de Bifungites, ficando classificado como Bifungites isp.; (iii) verificou-se que o exemplar DGM 4945-I apresenta uma classificação problemática, pois suas características, provavelmente em decorrência de uma preservação deficiente, correspondem apenas parcialmente à diagnose de Crossopodia, sendo então duvidosamente atribuído a este icnogênero e referenciado como Crossopodia? maranhensis (Kegel, 1966); (iv) os exemplares DGM 4434-I e DGM 4435-I, anteriormente descritos como Crossopodia estrellada por Kegel (1966), foram identificados como Phycosiphon incertum FischerOoster, 1858, por apresentarem morfologia característica desta última icnoespécie; (v) o exemplar DGM 4944-I descrito por Kegel (1966) como Spirophyton sp. foi reclassificado como Zoophycos isp., face à revisão icnotaxonômica do icnogênero Zoophycos realizada por Olivero (2007) que incluiu a designação Spirophyton como um de seus sinônimos. O exemplar DGM 4923-I, descrito por Kegel (1966) como Neoskolithos picosensis, foi mantido de acordo com sua classificação original. O exemplar descrito por Kegel (1961) como rasto de ?Homalonotus não foi discutido neste trabalho pelo fato do exemplar de número DGM 5424-I não ter sido localizado nas coleções do Museu de Ciências da Terra/ DNPM/RJ.

\section{AGRADECIMENTOS}

À R.C.T. Cassab e ao Departamento Nacional da Produção Mineral (DNPM), do Rio de Janeiro, pela permissão e acesso ao material descrito neste trabalho. A J.H.G. Melo pelas informações referentes aos poços da Petrobras e por sugestões que resultaram no aprimoramento do texto. Os autores agradecem ao CNPq pelo apoio financeiro (401762/2010-6 e 401763/2010-2). ACSF agradece ao CNPq pela bolsa de 
produtividade em pesquisa e LCMOP pela bolsa de doutorado. Este trabalho foi uma contribuição ao Simpósio LatinoAmericano de Icnologia - SLIC 2010.

\section{REFERÊNCIAS}

Agostinho, S.M.O. \& Fernandes, A.C.S. 2001. Avaliação do conteúdo icnológico da Formação Pimenteira, Devoniano do Parnaíba. Revista Brasileira de Paleontologia, 2:154.

Agostinho, S. \& Valença, L.M.M. 1999. Icnofósseis da região de Pimenteiras, PI, Formação Pimenteira, Devoniano da Bacia do Parnaíba. In: CONGRESSO BRASILEIRO DE PALEONTOLOGIA, 16, Crato. Boletim de Resumos, Crato, SBP e URCA, p. 11.

Agostinho, S.; Campelo, F.M.C.A.; Melo, K.J.V.; Viana, M.S.S. \& Fernandes, A.C.S. 2001. Perfil estratigráfico da Formação Pimenteira (Devoniano da Bacia do Parnaíba) no leito do rio Sambito (Pimenteiras-PI). In: CONGRESSO BRASILEIRO DE P\&D EM PETRÓLEO E GÁS, 1, Natal. Resumos, Natal, UFRN/SBQ, p. 56.

Agostinho, S.; Corrêa, L.M.S.A. \& Fernandes, A.C.S. 2003a. Os icnofósseis da Formação Pimenteira (Devoniano da Bacia do Parnaíba) no Município de Miranorte, Estado do Tocantins. In: CONGRESSO BRASILEIRO DE PALEONTOLOGIA, 18, Brasília. Boletim de Resumos, Brasília, SBP, p. 34.

Agostinho, S.; Viana, M.S.S. \& Fernandes, A.C.S. 2003b. Um novo registro do icnogênero Bifungites na Formação Pimenteira (Devoniano), Bacia do Parnaíba, Brasil. In: CONGRESSO BRASILEIRO DE PALEONTOLOGIA, 18, Brasília. Boletim de Resumos, Brasília, SBP, p. 35.

Agostinho, S.; Viana, M.S.S. \& Fernandes, A.C.S. 2004. Duas novas icnoespécies de Bifungites Desio, 1940 na Formação Pimenteira, Devoniano da Bacia do Parnaíba, Brasil. Arquivos do Museu Nacional, 62:519-530.

Alpert, S.P. 1974. Systematic review of the genus Skolithos. Journal of Paleontology, 48:661-669.

Assis, J.F.P. \& Fernandes, A.C.S. 1980. A ocorrência de Bifungites Desio, 1940 na Formação Pimenteiras, Devoniano da Bacia do Maranhão. Anais da Academia Brasileira de Ciências, 52:335-338.

Barroso, E.M.; Barroso, J.A.; Horta, A.E.D.G. \& Carvalho, I.S. 2008. Retrospectiva dos 50 Anos da Geologia na UFRJ e Olhar Crítico para o Futuro. Anuário do Instituto de Geociências, 31:9-23.

Borghi, L.; Metelo, C.M.S. \& Fernandes, A.C.S. 1999. Novas ocorrências de icnofósseis na Formação Pimenteira (Devoniano, Bacia do Parnaíba) no Município de São Raimundo Nonato, Estado do Piauí. Anais da Academia Brasileira de Ciências, 71:151-152.

Brito, I.M. 1977. Ocorrência de bióglifos no Devoniano Inferior do Município de Tocantínia, Goiás. Anais da Academia Brasileira de Ciências, 49:461-464.

Bromley, R.G. 1996. Trace Fossils - Biology, Taphonomy and Applications. $2^{\mathrm{a}}$ ed., Londres, Chapman and Hall, $361 \mathrm{p}$.

Buatois, L.A. \& Mángano, M.G. 1996. Icnologia de ambientes continentales: problemas y perspectivas. Asociación Paleontológica Argentina, Publicación Especial, 4:5-30.

Buatois, L.A. \& Mángano, M.G., 2004. Animal-substrate interactions in freshwater ecosystems: applications of ichnology in facies and sequence stratigraphic analysis of fluvio-lacustrine successions. In: D. McIlroy (ed.) The application of ichnology to palaeoenvironmental and stratigraphic analysis, Geological Society of London Special Publication 228, p. 311-333.
Campanha, V.A. 1974. Caracterização ichnofaciológica do Membro Picos, Formação Pimenteiras, na região de São Miguel do Tapuio. Piaú. Anais da Academia Brasileira de Ciências, 46:593-600.

Campelo, F.M.A.C. \& Viana, M.S.S. 2003. A palaeoenvironmental interpretation of the Pimenteira Formation, Parnaíba Basin, Northeast Brazil, based on ichnofossils. In: LATINAMERICAN CONGRESS OF SEDIMENTOLOGY, 3, Belém. Abstracts, Belém, MPEG/UFPA, p. 172-173.

Caputo, M.V. 1984. Stratigraphy, tectonics, paleoclimatology and paleogeography of northern basins of Brazil. Universidade da Califórnia, Tese de Doutorado, $583 \mathrm{p}$.

Caputo, M.V.; Iannuzzi R. \& Fonseca V.M.M. 2005. Bacias sedimentares brasileiras: Bacia do Parnaíba. Phoenix, 81:1-6.

Carozzi, A.V.; Falkenhein, F.U.H.; Carneiro, R.G.; Esteves, F.R. \& Contreiras, C.J.A. 1975. Análise ambiental e evolução tectônica sinsedimentar da seção siluro-eocarbonífera da Bacia do Maranhão. Cenpes/Petrobrás, Série Ciência-Técnica-Petróleo, 7:15-27.

Carvalho, M.G.P. 1995. Trilobitas do Devoniano da Bacia do Parnaiba. Programa de Pós-graduação em Geologia, Universidade Federal do Rio de Janeiro, Tese de Doutorado, $132 \mathrm{p}$.

Della Fávera, J.C. 1990. Tempestitos da Bacia do Parnaíba: um ensaio holístico. Programa de Pós-graduação em Geologia, Universidade Federal do Rio Grande do Sul, Tese de Doutorado, $243 \mathrm{p}$.

Fernandes, A.C.S. \& Fonseca. V.M.M. 2005. A contribuição de Ignácio Machado Brito à paleoicnologia brasileira. Arquivos do Museu Nacional, 63:619-624.

Fernandes, A.C.S.; Agostinho, S. \& Viana, M.S.S. 2003. O icnogênero Bifungites e sua nova ocorrência na Formação Pimenteira (Devoniano), Bacia do Parnaíba, Brasil. Ciências da Terra (Universidade Nova de Lisboa), n. especial 5:A72-A75.

Fernandes, A.C.S.; Borghi, L.; Carvalho, I.S. \& Abreu, C.J. 2002. Guia dos Icnofósseis de Invertebrados do Brasil. Rio de Janeiro, Interciência, $260 \mathrm{p}$.

Ferreira, C.S. \& Fernandes, A.C.S. 1983. Notícias sobre alguns icnofósseis da Formação Pimenteira, Devoniano no Estado de Goiás. Anais da Academia Brasileira de Ciências, 55:140.

Fillion, D. \& Pickerill, R.K. 1990. Ichnology of the Upper Cambrian? to Lower Ordovician Bell Island and Wabana groups of eastern Newfoundland, Canada. Palaeontographica Canadiana, 7:1-119.

Fonseca, V.M.M. \& Melo, J.H.G. 1987. Ocorrência de Tropiloleptos carinatos (Conrad) (Brachiopoda, Orthida) na Formação Pimenteira, e sua importância paleobiogeográfica. In: CONGRESSO BRASILEIRO DE PALEONTOLOGIA, 10, 1987. Anais, Rio de Janeiro, SBP, 2:505-537.

Gérard, J.R.F. \& Bromley, R.G. 2008. Ichnofabrics in clastic sediments: applications to sedimentological core studies. Madrid, IBERGRAPHI, 100 p.

Góes, A.M.O \& Feijó, F.J. 1994. Bacia do Parnaíba. Boletim de Geociências da Petrobras, 8:58-60.

Grahn, Y.; Melo, J.H.G. \& Steemans, P. 2005. Integrated chitinozoan and miospore zonation of the Serra Grande Group (Silurianlower Devonian), Parnaíba Basin, northeast Brazil. Revista Española de Micropaleontología, 37:183-204.

Häntzschel, W. 1975. Trace fossils and problematica. In: C. Teichert (ed.) Treatise on Invertebrate Paleontology, Part W, Miscellanea, Supplement I, Geological Society of America and University of Kansas Press, 269 p.

Kegel, W. 1953. Contribuição para o estudo do Devoniano da Bacia do Parnaíba. Rio de Janeiro, Departamento Nacional da 
Produção Mineral, Divisão de Geologia e Mineralogia, 48 p. (Boletim 14).

Kegel, W. 1957. Sobre vermes recentes e fósseis. Rio de Janeiro, Departamento Nacional da Produção Mineral, Divisão de Geologia e Mineralogia, 11 p. (Notas Preliminares e Estudos $100)$.

Kegel, W. 1961. Rasto de um trilobita (?Homalonotus) do Devoniano Inferior do Piauí. Anais da Academia Brasileira de Ciências, 33:163-167.

Kegel, W. 1965a. Rastos de Bilobites no Devoniano Médio do Piauí. Rio de Janeiro, Departamento Nacional da Produção Mineral, Divisão de Geologia e Mineralogia, p. 55-56 (Avulso 40).

Kegel, W. 1965b. Rastos de Bilobites no Devoniano Médio do Piauí. Rio de Janeiro, Departamento Nacional da Produção Mineral, Divisão de Geologia e Mineralogia, 11 p. (Notas Preliminares e Estudos 122).

Kegel, W. 1966. Rastos do Devoniano da Bacia do Parnaíba. Rio de Janeiro, Departamento Nacional da Produção Mineral, Divisão de Geologia e Mineralogia, 32 p. (Boletim 233).

Melo, J.H.G. 1985. A Província Malvinocáfrica no Devoniano do Brasil: estado atual dos conhecimentos. Programa de Pósgraduação em Geologia, Universidade Federal do Rio de Janeiro, Mestrado em Geologia, 1357 p.

Muniz, G.C.B. 1979. Os ichnofósseis e sua importância nas ciências geológicas. Estudos e Pesquisas, 3:23-29.

Muniz, G.C.B. 1981a. A fácies Arenito Cacimba (Formação Inajá): detalhes estratigráficos e novas observações ichnológicas. Estudos Pesquisas, 4:7-15.

Muniz, G.C.B. 1981b. Uma inusitada população de Rusophycus na Formação Longá, Devoniano, no estado do Piauí. In: SIMPÓSIO DE GEOLOGIA DO NORDESTE, 10, Recife. Atas, Recife, SBG, p. 262-264.

Muniz, G.C.B. 1982. Ichnofósseis Devonianos da Formação Longá, no Estado do Piauí. In: CONGRESSO BRASILEIRO DE GEOLOGIA, 32, 1982. Anais, Salvador, SBG, 4:1305-1316.

Nascimento, M.C.; Moura, C.R.; Campelo, F.M.A.C.; Agostinho, S. \& Viana, M.S.S. 2003. Paleoenvironmental significance of trilobite traces in the Parnaíba Basin, NE of Brazil. In: LATINAMERICAN CONGRESS OF SEDIMENTOLOGY, 3, 2003. Abstracts, Belém, MPEG e UFPA, p. 176-177.

Netto, R.G. 2007. Skolithos-dominated piperock in non-marine environments: an example from the Triassic Caturrita Formation, southern Brazil. In: R.G. Bromley; L.A. Buatois; M.G. Mángano; J.F. Genise \& R.N. Melchor (orgs.) Sediment- organism interactions: a multifaceted ichnology, SEPM Special Publication 88, p. 109-121.

Olivero, D. 2007. Zoophycos and the role of type specimens in ichnotaxonomy. In: W. Miller III (ed.) Trace Fossils - concepts, problems, prospects, Elsevier, p. 219-231.

Ponciano, L.C.M.O. \& Della Fávera, J.C. 2009. Flood-dominated fluvio-deltaic system: a new depositional model to Cabeças Formation, Parnaíba Basin, Piauí, Brazil. Anais da Academia Brasileira de Ciências, 81:769-780.

Rodrigues, A.S. 2001. Revisão dos icnofósseis descritos por Wilhelm Kegel para as formações Cabeças e Longá (Devoniano Médio e Superior da Bacia do Parnaíba). In: JORNADA DE INICIAÇÃO CIENTÍFICA, 23, 2001. Resumos, Rio de Janeiro, UFRJ, p. 124.

Santos, M.E.C.M. \& Carvalho, M.S.S. 2009. Paleontologia das bacias do Parnaíba, Grajaú e São Luís. Rio de Janeiro, Companhia de Pesquisa de Recursos Minerais/Serviço Geológico do Brasil, 226 p.

Seilacher, A. 2003. Arte fóssil. Divulgações do Museu de Ciências e Tecnologia, UBEA/PUCRS, Publicação especial EDIPUCRS, 86 p.

Silva, S.M.O.A. 1999. Icnofósseis da Formação Pimenteira, Devoniano da Bacia do Parnaíba, Municipio de Pimenteiras, Piauí. Programa de Pós-Graduação em Geociências, Universidade Federal de Pernambuco, Dissertação de Mestrado, 46 p.

Turner, B.R. \& Benton, M.J. 1983. Paleozoic trace fossils from the Kufra Basin, Libya. Journal of Paleontology, 57:447-460.

Vaz, P.T.; Rezende, N.G.A.M.; Wanderley Filho, J.R. \& Travassos, W.A.S. 2007. Bacia do Parnaíba. Boletim de Geociencias da Petrobras, 15:253-263.

Wetzel, A. \& Bromley, R.G. 1994. Phycosiphon incertum revisited: Anconichnus horizontalis is its junior subjective synonym. Journal of Paleontology, 68:1396-1402.

Young, C.G.K. \& Borghi, L. 2003. An event of forced regression in the Pimenteira Formation (Devonian, Parnaíba Basin, Brazil). In: LATINAMERICAN CONGRESS OF SEDIMENTOLOGY, 3, 2003. Abstracts, Belém, MPEG/UFPA, p. 168-170.

Young, C.G.K.; Borghi, L. \& Fernandes, A.C.S. 2002. Icnofósseis na Formação Pimenteira (Devoniano, Bacia do Parnaíba) em testemunhos de sondagem. In: PALEO 2002, Rio de Janeiro, Paleontologia em Destaque, 40:21.

Received in January, 2011; accepted in February, 2012. 